\title{
Control of electronic conduction at an oxide heterointerface using surface polar adsorbates
}

Yanwu Xie ${ }^{1,2}$, Yasuyuki Hikita², Christopher Bell ${ }^{2} \&$ Harold Y. Hwang ${ }^{1,2}$

The interface between $\mathrm{LaAlO}_{3}$ and $\mathrm{SrTiO}_{3}$ possesses a range of intriguing properties, notably a proposed connection between the surface state of the $\mathrm{LaAlO}_{3}$ and the conductivity buried in the $\mathrm{SrTiO}_{3}$. Here we study the effect of the surface adsorption of a variety of common laboratory solvents on the conductivity at the interface between $\mathrm{LaAlO}_{3}$ and $\mathrm{SrTiO}_{3}$. We show that the application of chemicals such as acetone, ethanol, and water can induce a large change in the conductivity, and, in particular, an insulator to metal transition around the critical $\mathrm{LaAlO}_{3}$ thickness. This phenomenon is observed only for polar solvents. These data provide experimental evidence for a general polarization-facilitated electronic transfer mechanism.

\footnotetext{
${ }^{1}$ Department of Advanced Materials Science, University of Tokyo, Kashiwa, Chiba 277-8561, Japan. ${ }^{2}$ Department of Applied Physics and Stanford Institute for Materials and Energy Science, Stanford University, Stanford, California 94305, USA. Correspondence and requests for materials should be addressed to H.Y.H. (email: hyhwang@stanford.edu).
} 
$\mathrm{T}$ he transfer of electrons between a solid surface and adsorbed atomic or molecular species is fundamental in natural and synthetic processes, being at the heart of most catalytic reactions and many sensors. In special cases, metallic conduction can be induced at the surface of, for example, Si-terminated $\mathrm{SiC}^{1}$, or mixedterminated $\mathrm{ZnO}^{2}$, in the presence of a hydrogen adlayer. Generally, only the surface atoms are significantly affected by adsorbates. However, remotely changing electronic states far from the adsorbed layer is possible, if these states are electrostatically coupled to the surface. Here we show that the surface adsorption of common solvents such as acetone, ethanol, and water can induce a large change in the conductivity at the buried interface between $\mathrm{SrTiO}_{3}$ substrates and $\mathrm{LaAlO}_{3}$ thin films s-8, and an adsorbate induced insulator to metal transition was observed when the thickness of $\mathrm{LaAlO}_{3}$, $d_{\mathrm{LAO}}$, is around 3 unit cells (uc). This phenomenon is observed only for polar solvents. Our result provides experimental evidence that adsorbates at the $\mathrm{LaAlO}_{3}$ surface induce accumulation of electrons at the $\mathrm{LaAlO}_{3} / \mathrm{SrTiO}_{3}$ interface, suggesting a general polarizationfacilitated electronic transfer mechanism, which can be adapted for use in a range of oxide-based devices.

The intense interest in the $\mathrm{LaAlO}_{3} / \mathrm{SrTiO}_{3}$ interface ${ }^{3-22}$ has led to several recent experimental observations that suggest a close relationship between the interface and the $\mathrm{LaAlO}_{3}$ surface. These include the use of conducting atomic force microscopy probes to toggle a metal insulator transition ${ }^{10-12}$ through the writing of surface charge ${ }^{12,13}$, and the use of capping layers of $\mathrm{SrTiO}_{3}$ (ref. 14) or $\mathrm{SrCuO}_{2}$ (ref. 15) to alter the interface conductivity. The origin of these effects, and indeed the interface conductivity itself, remains in debate ${ }^{4,16-22}$. The transfer of electrons from the surface to the interface, to reconcile the polar discontinuity between the neutral $\{100\}$ layers in $\mathrm{SrTiO}_{3}$ and the charged layers in $\{100\} \mathrm{LaAlO}_{3}$, is one model that naturally connects the electronic states of the interface and $\mathrm{LaAlO}_{3}$ surface $^{4,18-22}$. However, despite these concepts and recent experimental progress, the effect of surface adsorbates has not been investigated. This issue is addressed in the present work. We have found that the exposure of $\mathrm{LaAlO}_{3} / \mathrm{SrTiO}_{3}$ samples to a polar solvent can increase the sheet carrier density, $n_{2 d}$, by more than $2 \times 10^{13} \mathrm{~cm}^{-2}$, representing a change of the same order as the total charge density typical in this system $^{5-8}$. Compared with the strong perturbations associated with other surface processes, that is, the extremely strong local electric field produced by the conducting atomic force microscopy probes ${ }^{10-13}$, or the structural variation by introducing capping layers grown at high temperatures ${ }^{14,15}$, the changes associated with room-temperature treatment using these solvents would naively be expected to be small. However, such processes result in a surprisingly large modulation of $n_{2 d}$, revealing a dramatic surface-interface coupling.

\section{Results}

Transport characterization. The fabrication of the conducting $\mathrm{LaAlO}_{3} / \mathrm{SrTiO}_{3}$ interfaces and the surface adsorption process (SAP) are described in the Methods. As shown in Figure 1a, labels 1 \& 2 , a SAP using acetone increased $n_{2 d}$ from $\sim 1 \times 10^{13} \mathrm{~cm}^{-2}$ to more than $3 \times 10^{13} \mathrm{~cm}^{-2}$ for a $d_{\mathrm{LAO}}=10 \mathrm{uc}$ sample, over a wide temperature range $(2 \mathrm{~K} \leq T \leq 300 \mathrm{~K})$. This remarkable modulation suggests that the acetone molecules have been adsorbed on the $\mathrm{LaAlO}_{3}$ surface and, in turn, change the electronic states at the buried interface. Heating the sample at $T=380 \mathrm{~K}$ in a moderate vacuum $\left(<10^{3} \mathrm{~Pa}\right.$, with a helium background) for several hours produced negligible conduction change (not shown). However, the original sample state was recovered after heating at an elevated temperature of $653 \mathrm{~K}$, evidenced by the decrease in $n_{2 d}$ (Fig. 1a, label 3) and the subsequent increase after another SAP using water (Fig. 1a, label 4). These facts indicate that the surface adsorption is reversible but strong, not a physical adsorption driven by the relatively weak van der Waals force, and thus likely involves electron transfer. We note that as a function of $n_{2 d}$, the qualitative shape of the $n_{2 d}(T)$ data at
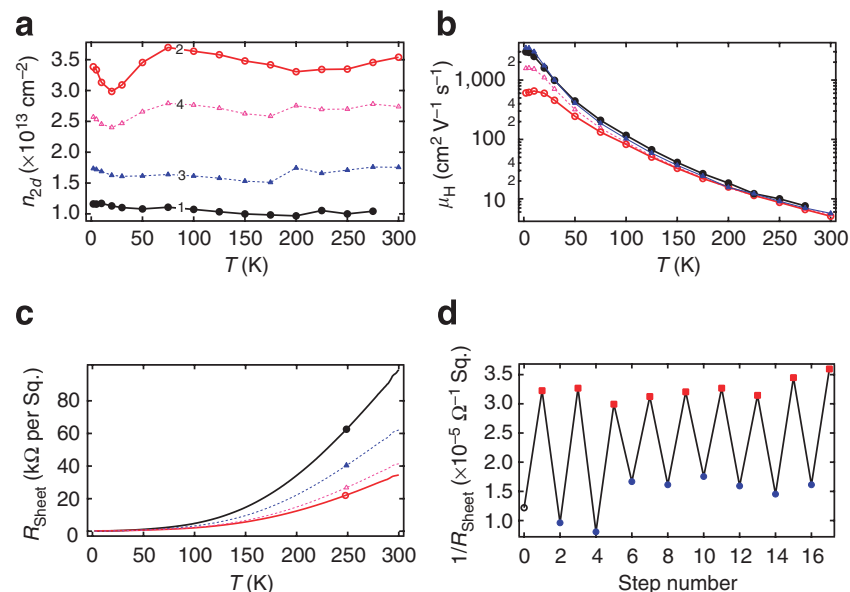

Figure 1 | Transport characterization. Temperature dependence of (a) Sheet carrier density, $n_{2 d^{\prime}}$ (b) Hall mobility, $\mu_{H}$, and (c) Sheet resistance, $R_{\text {Sheet }}$ in different states of a $d_{\mathrm{LAO}}=10$ uc $\mathrm{LaAlO}_{3} / \mathrm{SrTiO}_{3}$ sample. The black closed circles represent the as-grown sample before experiencing any SAP. The red open circles represent the sample after an acetone SAP. The blue closed triangles represent the sample after heating at $T=663 \mathrm{~K}$ in an $\mathrm{O}_{2}$ pressure of $1.33 \times 10^{-2} \mathrm{~Pa}$ for $5 \mathrm{~h}$. The pink open triangles represent the sample after a water SAP. The processing and measurement sequence follows the labelled order in (a). (d) Room-temperature conductivity, $1 / R_{\text {sheet }}$ of a $d_{\mathrm{LAO}}=10$ uc $\mathrm{LaAlO}_{3} / \mathrm{SrTiO}_{3}$ sample that was repeatedly processed by water SAP (red closed squares) and recovered by heating at $573 \mathrm{~K}$ in an oxygen flow for $3 \mathrm{~h}$ (blue closed circles). The black open circle represents the as-grown sample.

low temperatures is quite different. This is likely associated with the complex temperature and electric-field dependencies of $\mathrm{SrTiO}_{3}$, leading to a non-trivial variation of the confining potential as a function of $n_{2 d}$, as discussed elsewhere ${ }^{23}$.

Accompanying the $n_{2 d}$ increase, a striking reduction in the Hall mobility, $\mu_{\mathrm{H}}$, from $3,000 \mathrm{~cm}^{2} \mathrm{~V}^{-1} \mathrm{~s}^{-1}$ to $600 \mathrm{~cm}^{2} \mathrm{~V}^{-1} \mathrm{~s}^{-1}$ (T=2K) is observed after the acetone SAP (Fig. 1b). $\mu_{\mathrm{H}}$ decreases quickly with increasing temperature, with a relatively small room temperature value of $\mu_{\mathrm{H}} \sim 6 \mathrm{~cm}^{2} \mathrm{~V}^{-1} \mathrm{~s}^{-1}$ due to the dominance of phonon scattering. At this temperature, $\mu_{\mathrm{H}}$ also has a very weak dependence on $n_{2 d}$, thus the conductivity $1 / R_{\text {sheet }}$ is a reasonable and convenient index of $n_{2 d}$ (Fig. 1a, c), and we will use it hereafter to characterize the effect of the SAP process on $n_{2 d}$ for a variety of common solvents. To study the repeatability of the adsorption/desorption processes, we repeatedly performed water $\mathrm{SAP}$ on a $\mathrm{LaAlO}_{3} / \mathrm{SrTiO}_{3}$ sample followed by a heating step at $573 \mathrm{~K}$ in an oxygen flow, to reset the system (Fig. 1d). The sample switches reproducibly between a high-conductivity state after SAP and a low-conductivity state after heating.

Effect of SAP using different solvents. To explore the origin of the SAP-induced $n_{2 d}$, we have studied the effect of SAP using a variety of solvents with $d_{\mathrm{LAO}}=10 \mathrm{uc}$ samples. These solvents can be classified into three categories: non-polar, polar aprotic (no dissociable $\mathrm{H}^{+}$), and polar protic (dissociable $\mathrm{H}^{+}$). The results are summarized in Figure 2. Clearly, SAP using non-polar solvents produces no significant modulation in $1 / R_{\text {sheet }}$ and by extension, $n_{2 d}$. SAP using a relatively weak polar solvent such as ethyl acetate produces a slight increase in $n_{2 d}$, whereas using more polar solvents produces a much larger increase, independent of whether the solvents are aprotic or protic. In Figure 2 , we also show that $\Delta\left(1 / R_{\text {Sheet }}\right)$ is well scaled with the molecular dipole moment divided by the molecular volume. These results suggest that the polar nature of solvents has a key role in determining the magnitude of the observed changes for the different solvents. In the context of an electrostatic mechanism, the scaling of 


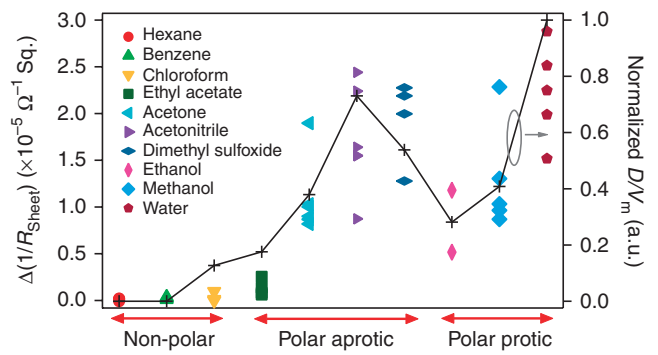

Figure 2 | Effect of SAP using different solvents. The difference in $1 / R_{\text {sheet }}$ after and before SAP, $\Delta\left(1 / R_{\text {sheet }}\right)$, reflects the change in $n_{2 d}$ at room temperature. A clear increase in $\Delta\left(1 / R_{\text {sheet }}\right)$ is observed after SAP using polar solvents, independent of their aprotic or protic character. $\Delta\left(1 / R_{\text {sheet }}\right)$ is reasonably scaled with the molecular dipole moment $(D)$ of each solvent divided by the corresponding molecular volume $\left(V_{m}\right)$ (crosses). The line is a guide to the eye. In each category, the solvents are ordered from left to right by increasing polarizability. At least two samples were used for each solvent and all samples were $d_{\mathrm{LAO}}=10 \mathrm{uc}$ and in the as-grown state before SAP.

the change with the dipole moment density would be reasonable, as the strength of the effect should be related to not only the molecular dipole moment, but also the areal density of the molecules on the surface.

Comparison between SAP and adsorption of solvent vapour. Further insights into the origin of the SAP-induced $n_{2 d}$ can be made by comparing the effect of SAP with the effect of adsorption from a solvent vapour. It is well known that a thin layer of water coats all hydrophilic surfaces under ambient conditions ${ }^{24}$. Until now the adsorption of water on $\mathrm{LaAlO}_{3}$, particularly on the $\mathrm{AlO}_{2}$-terminated $\mathrm{LaAlO}_{3}$ surface, has been scarcely studied. A water-dipping study showed that, whereas $\mathrm{La}_{2} \mathrm{O}_{3}$ is highly hygroscopic, $\mathrm{LaAlO}_{3}$ only reacts weakly with water ${ }^{25}$. In contrast, the adsorption of water on $\mathrm{Al}_{2} \mathrm{O}_{3}$ is well understood. It is known that $\mathrm{Al}_{2} \mathrm{O}_{3}$ adsorbs 1 monolayer of water when the relative humidity $(\mathrm{RH})$ is $\sim 35 \%$, and more than 5 monolayers for $\mathrm{RH}>70 \%$ (refs 26,27 ). Thus, although these studies cannot be trivially extrapolated to the current heterostructures, we expect a thin layer of water with full coverage will coat the $\mathrm{AlO}_{2}$-terminated $\mathrm{LaAlO}_{3}$ surface when the $\mathrm{LaAlO}_{3} / \mathrm{SrTiO}_{3}$ sample is exposed to $\mathrm{RH}$ $>70 \%$ air at room temperature. Such a surface layer has also been inferred from charge writing experiments ${ }^{28}$. Nonetheless, despite the existing water layer, we can still modulate the conductivity using the macroscopically large volumes of solvent in the SAP process.

Figure $3 \mathrm{a}$ shows that the conductivity of $\mathrm{LaAlO}_{3} / \mathrm{SrTiO}_{3}$ is still sensitive to water SAP using liquid even after exposing it to watersaturated atmosphere for several hours. A similar result was found when acetone was used (Fig. 3b). From these results, we can conclude that the thickness of adsorbate layer also has an important role in the SAP effect, as the SAP with liquid is expected to produce a thicker adsorbed solvent film compared with the vapour experiments.

$\mathrm{LaAlO}_{3}$ thickness dependence. A key feature of the $\mathrm{LaAlO}_{3} / \mathrm{SrTiO}_{3}$ interface is that there exists a critical $\mathrm{LaAlO}_{3}$ thickness, $d_{\text {LAO }} \sim 4 \mathrm{uc}$, below which the interface is insulating ${ }^{5}$. It is instructive to see the dependence of the effect of SAP on the $\mathrm{LaAlO}_{3}$ thickness. As shown in Figure $4 \mathrm{a}$, for $d_{\mathrm{LAO}}=2 \mathrm{uc}$ sample and bare $\mathrm{SrTiO}_{3}$ substrate, no modulation in the conductivity is observed. For $d_{\mathrm{LAO}}=3 \mathrm{uc}$, the as-grown sample is insulating and the acetone SAP drives the sample from an insulating state to a conducting state. In this case, the absolute modulation in the carrier density, as indexed by $\Delta\left(1 / R_{\text {Sheet }}\right)$, is smaller than that in the thicker samples, but the sensitivity, defined as the ratio of the conductivity change with respect to that of the as-grown sample, is much larger. We speculate that, with suitable optimization, this large sensitivity may be exploited for sensor applications.
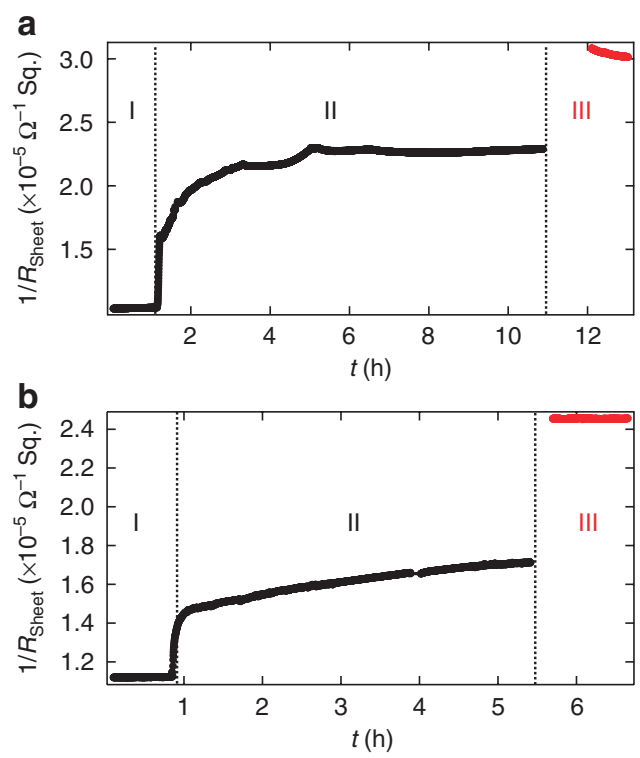

Figure 3 | Comparison between the effect of SAP and the effect of adsorption of solvent vapour. (a) Conductivity versus time plots for a sample exposed to (I) air of $\mathrm{RH}=30 \%$, (II) air near the saturation point of water, and (III) followed by a water SAP. (b) Conductivity versus time plots for a sample exposed to (I) air of $\mathrm{RH}=30 \%$, (II) air near the saturation point of acetone, and (III) followed by an acetone SAP. Note that surface adsorption of, for example, water, would have already taken place when the sample was kept in normal air. The exposure to the nearly saturated water (acetone) vapour for many hours should achieve a full and thicker coverage of adsorbates on the $\mathrm{LaAlO}_{3}$ surface. The samples are $d_{\mathrm{LAO}}=10 \mathrm{uc}$, and the measurements were performed at ambient environment. The irregular perturbations in (a) were caused by fluctuations in the local illumination and room temperature.

Figure $4 \mathrm{~b}$ shows the time-dependent $1 / R_{\text {sheet }}$, immediately after an acetone SAP, of $\mathrm{LaAlO}_{3} / \mathrm{SrTiO}_{3}$ samples of different $\mathrm{LaAlO}_{3}$ thicknesses. As $d_{\mathrm{LAO}}$ decreases, $1 / R_{\text {sheet }}$ tends to relax more quickly, indicating solvent desorption. This observation suggests that the surface adsorption is directly related to the nature of the $\mathrm{LaAlO}_{3}$ surface, or its polar character. We note that a somewhat analogous observation has previously been found for the charge stability on the $\mathrm{LaAlO}_{3}$ surface in the same system ${ }^{12}$.

\section{Discussion}

Next, we discuss the possible mechanisms that may explain these data. We exclude electrostatic attraction because the polar adsorbates themselves are charge-neutral as a whole and will not change the electrostatic boundary condition of $\mathrm{LaAlO}_{3} / \mathrm{SrTiO}_{3}$ without the transfer of electrons ${ }^{29}$. Oxygen vacancies in the $\mathrm{SrTiO}_{3}$ (ref. 16), atomic interdiffusion ${ }^{17}$, and an electronic reconstruction $^{4,18-20}$ have all been suggested to be the origin of the interfacial conductivity in the $\mathrm{LaAlO}_{3} / \mathrm{SrTiO}_{3}$ system. In the case of the SAP experiments, we exclude the first two of these as we assume that a SAP at room temperature does not cause significant atomic structure changes at the interface, and we focus on the electronic reconstruction mechanism.

As discussed in several papers ${ }^{19,20}$, and illustrated in Figure 5a, for an idealized $\mathrm{LaAlO}_{3}$ surface free from surface states, the valence band of $\mathrm{LaAlO}_{3}, E_{V}^{\mathrm{LAO}}$ is the electron source for the interface electrons. Here we assume no conductivity in the $\mathrm{LaAlO}_{3}$ film itself, and, for simplicity, do not consider other processes that do not affect the electrostatic state of the $\mathrm{LaAlO}_{3}$. An uncompensated potential, $V_{\text {Uncom }}$, across the $\mathrm{LaAlO}_{3}$ film of the order of the band gap of $\mathrm{SrTiO}_{3}$, 


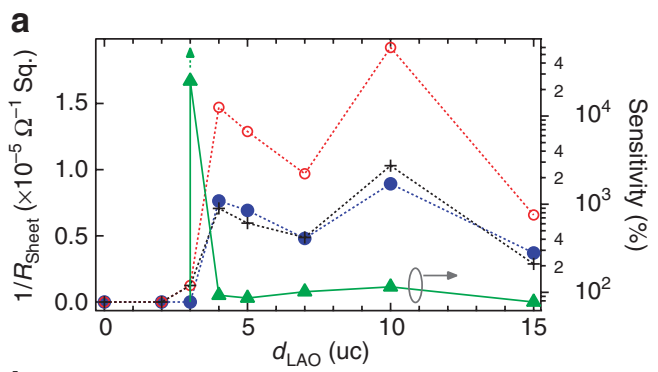

b

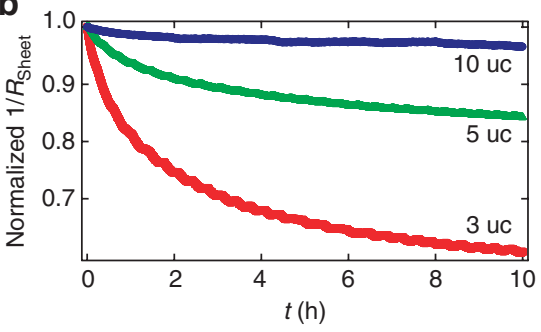

Figure 4 | $\mathrm{LaAlO}_{3}$ thickness dependence. (a) The effect of SAP on samples of different $\mathrm{LaAlO}_{3}$ thicknesses. The blue closed circles represent $1 / R_{\text {sheet }}$ of the as-grown samples. The red open circles represent $1 / R_{\text {sheet }}$ of the samples after an acetone SAP. The difference after and before acetone SAP, $\Delta\left(1 / R_{\text {sheet }}\right)$, is represented by black crosses. Sensitivity (green triangles) was defined as a ratio between $\Delta\left(1 / R_{\text {sheet }}\right)$ and $1 / R_{\text {sheet }}$ (as grown). The green arrow indicates the measurement limit. The $d_{\mathrm{LAO}}=0 \mathrm{uc}$ sample is a bare $\mathrm{SrTiO}_{3}$ substrate. The acetone SAP triggers a transition from an insulating state to a conducting state for a $d_{\mathrm{LAO}}=3 \mathrm{uc}$ sample. (b) The time dependence of $1 / R_{\text {sheet }}$ after acetone SAP for $d_{\text {LAO }}=3,5$, and 10 uc samples. All measurements were performed at ambient environment. The small perturbations in the data, notably on the 3 uc curve, were caused by small fluctuations in the room temperature. Lines are guides for the eye.

$E_{g}^{\text {STO }}$ is needed to align the chemical potential over the whole structure. A simple electrostatic consideration shows that

$$
V_{\text {Uncom }}=\frac{e\left(\frac{\sigma_{0}}{2}-\sigma_{\text {Inter }}\right)}{\varepsilon_{\mathrm{LAO}}} d_{\mathrm{LAO}} \text {, }
$$

where $\sigma_{0}$ is the charge density of one atomic layer, $\sigma_{\text {Inter }}$ is the total sheet electron density at the interface, $e$ is the unit charge, and $\varepsilon_{\mathrm{LAO}}$ is the dielectric constant of $\mathrm{LaAlO}_{3}$. In reality, $\sigma_{\text {Inter }}$ is larger than $n_{2 d}$ because of the trapping of some electrons at the interface ${ }^{19}$. Immediately, we find that

$$
\sigma_{\text {Inter }}=\frac{\sigma_{0}}{2}-\frac{\varepsilon_{\mathrm{LAO}}}{e d_{\mathrm{LAO}}} V_{\mathrm{Uncom}}
$$

This simple relationship predicts the same thickness dependence of $\sigma_{\text {Inter }}$ as several more detailed theoretical calculations ${ }^{20,30}$, noting that $V_{\text {Uncom }}$ is fixed for an ideal surface. In the work presented here, $d_{\text {LAO }}$ is fixed, and the SAP induced change in $n_{2 d}$ is thus associated with a reduction in $V_{\text {Uncom. }}$.

We note that there are several possibilities of reduction of $V_{\text {Uncom }}$. In practice, all samples have surface states associated with inevitable defects, surface reconstructions, surface adsorbates and so on. If these surface states are within the bandgap of $\mathrm{LaAlO}_{3}$, they can replace $E_{V}{ }^{\mathrm{LAO}}$ as more energetically favourable electron sources, where a relatively smaller $V_{\text {Uncom }}$ is required to dope electrons into the $\mathrm{SrTO}_{3}$. As far as surface adsorbates are concerned, they may interact with surface atoms or oxygen defects, and cause complex surface states. Two recent theoretical papers have suggested that the dissociation of hydrogen ${ }^{21}$ and redox reactions ${ }^{22}$ on the $\mathrm{LaAlO}_{3}$ sur- a

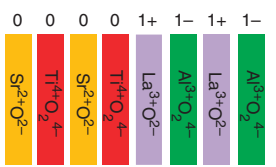

b
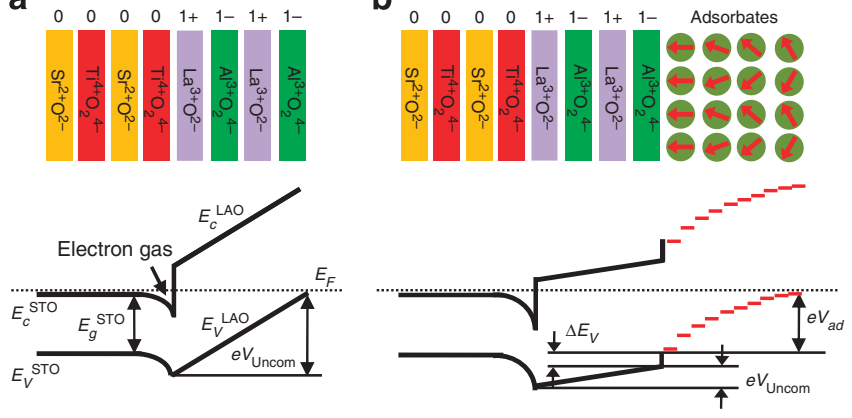

Figure 5 | Sketches of band diagrams and electron transfer mechanisms. (a) Idealized surface. Electrons transfer from $E_{V}{ }^{\mathrm{LAO}}$ of the $\mathrm{LaAlO}_{3}$ surface to the conduction band of $\mathrm{SrTiO}_{3}, E_{C}^{\text {STO }}$, near the interface. $e V_{\text {Uncom }}$ roughly equals to $E_{g}^{\text {STO }}$. (b) Surface with aligned polar adsorbates. Electrons transfer from the surface adsorbates to $E_{C}{ }^{\text {STO }}$ near the interface. The built-in potential across the adsorbates, $V_{\text {ad }}$, effectively reduces $V_{\text {uncom }}$ across the $\mathrm{LaAlO}_{3}$ layer. In both $(\mathbf{a}, \mathbf{b})$ bulk stoichiometry and valences are shown for simplicity.

face as possible electron sources. In both cases, $V_{\text {Uncom }}$ is reduced compared with that of the idealized surface. The role of water on the $\mathrm{LaAlO}_{3}$ surface has also been recently stressed in theoretical calculations by S. Hellberg (personal communication). However, we caution that the observed accumulation of electrons by SAP cannot be fully explained by adsorbate-induced surface states alone, because the polar character of the adsorbates and their thickness were found to have a vital role in our experimental results.

With the above picture in mind, we can interpret our results as follows: the strong polarization in the $\mathrm{LaAlO}_{3}$ layer aligns to a degree the polar adsorbates nearest the surface, with a decaying trend as we move away into the adsorbate layer. As shown schematically in Figure $5 b$, this leads to an electrostatic potential across the adsorbate layer, $V_{\text {ad }}$, quite similar to the built-in potential in the polar $\mathrm{LaAlO}_{3}$. As a result, electrons are sourced from the adsorbates instead of $E_{V} \mathrm{LAO}$ of the surface $\mathrm{LaAlO}_{3}$ to the $\mathrm{LaAlO}_{3} / \mathrm{SrTiO}_{3}$ interface, and $V_{\text {Uncom }}$ across the $\mathrm{LaAlO}_{3}$ is effectively decreased. $\sigma_{\text {Inter }}$ increases according to Equation (1), consistently explaining the observed SAP-induced accumulation of electrons at the $\mathrm{LaAlO}_{3} / \mathrm{SrTiO}_{3}$ interface. In this picture, the change in $\sigma_{\text {Inter }}$ is proportional to the value of $V_{\text {ad }}$ and the areal density of adsorbates. If we assume that the thickness of the adsorbate layer is the same for all solvents, and that all solvents are equally likely to transfer electrons it is simple to show that $\Delta \sigma_{\text {Inter }}$ will be proportional to $D / V_{m}$, consistent with the experimentally observed scaling relation between $\Delta\left(1 / R_{\text {Sheet }}\right)$ and $D / V_{m}$ (Fig. 2).

Our present observation is quite distinct from the proposed water-cycle mechanism that accounts for the modulation of interface conductivity by biased conductive scanning probe, because we achieve conductivity changes without actively dissociating water molecules ${ }^{28}$. We note that in principle the biased scanning probe will also change the physical distribution of the adsorbates, (for example, water), in addition to chemical changes, which may also contribute to the modulation in the interface conductivity in the charge writing experiments $\mathrm{s}^{10-13}$.

The observed close coupling between polar surface adsorbates and interface conductivity provides new insights into the origin of the electron gas at the $\mathrm{LaAlO}_{3} / \mathrm{SrTiO}_{3}$ interface, and demonstrates a new tuning parameter in controlling this state, which has the potential to be applied broadly in a wide range of novel oxide-based devices. These data also suggest that many of the conflicting (and often contradictory) studies of this popular system can be explained by this observation, that is, whether measurements are made 
in situ ${ }^{31}$, or after various surface exposures (for example, atmospheric water, or solvent cleaning $)^{32-34}$, the sensitive electronic structure of the interface may be dramatically altered. For example, the predicted electric field across the $\mathrm{LaAlO}_{3}$ layer ${ }^{18-20}$ was not observed in X-ray photoemission studies ${ }^{32}$ that may be explained consistently because the surface adsorbates (in particular atmospheric water) induced by the $e x$-situ processing reduce $V_{\text {Uncom }}$, or equally, the electrical field across the $\mathrm{LaAlO}_{3}$ layer. In contrast, in the $\mathrm{LaAlO}_{3} / \mathrm{SrTiO}_{3}$ superlattices of multiple interfaces, the interfaces relatively far away from the surface may not be significantly affected by adsorbates ${ }^{34}$.

\section{Methods}

Sample fabrication. Using optical lithography and lift-off, a six-contact Hall bar (central bar length $50 \mu \mathrm{m}$, width $10 \mu \mathrm{m}$ ) with amorphous $\mathrm{AlO}_{x}$ as a hard mask was patterned onto the atomically flat $\mathrm{TiO}_{2}$-terminated $\mathrm{SrTiO}_{3}(100)$ substrates. The $\mathrm{LaAlO}_{3}$ thin films were grown on the patterned substrates by pulsed laser deposition with the growth monitored by in-situ reflection high-energy electron diffraction. The conducting interfaces are confined within the Hall bar region. For the samples shown in this article, the growth was at $923 \mathrm{~K}$ in an $\mathrm{O}_{2}$ pressure of $1.33 \times 10^{-3} \mathrm{~Pa}$, after a pre-annealing at $1,223 \mathrm{~K}$ in an $\mathrm{O}_{2}$ pressure of $6.67 \times 10^{-4} \mathrm{~Pa}$ for half an hour. After deposition, there followed a post-annealing step at $873 \mathrm{~K}$ in an $\mathrm{O}_{2}$ pressure of $4 \times 10^{4} \mathrm{~Pa}$ for one hour. During deposition, the laser repetition was $1 \mathrm{~Hz}$ and the laser energy density was $0.6 \mathrm{Jcm}^{-2}$. The same phenomenology is found for samples grown in a variety of temperatures, oxygen pressures and laser conditions.

Electrical contact and measurement. The conducting interface was contacted by ultrasonic bonding with $\mathrm{Al}$ wire. The temperature-dependent sheet resistance, sheet carrier density and mobility were deduced from standard transport measurements using the patterned Hall bar. All transport measurements were carried out using a standard four-probe method. To test for the possibility of parallel conductivity through the adsorbate layer itself, contacts were made directly to the surface using silver epoxy. No significant surface conduction was detected both before and after SAP, clearly demonstrating that the conduction is at all times dominated by the $\mathrm{LaAlO}_{3} / \mathrm{SrTiO}_{3}$ interface.

Surface adsorption process. SAP was achieved by placing a drop of the liquid solvent on the sample surface at room temperature and blowing off all visible solvent within less than $10 \mathrm{~s}$ using dry nitrogen gas. Contacts were either left on the samples during the SAP, or reconnected afterwards; no significant difference in the conduction was observed by using either method.

\section{References}

1. Derycke, V. et al. Nanochemistry at the atomic scale revealed in hydrogeninduced semiconductor surface metallization. Nature Mater. 2, 253-258 (2003).

2. Wang, Y. et al. Hydrogen induced metallicity on the $\mathrm{ZnO}$ (1010) surface. Phys. Rev. Lett. 95, 266104 (2005).

3. Ohtomo, A. \& Hwang, H. Y. A high-mobility electron gas at the $\mathrm{LaAlO}_{3} / \mathrm{SrTiO}_{3}$ heterointerface. Nature 427, 423-426 (2004).

4. Nakagawa, N., Hwang, H. Y. \& Muller, D. A. Why some interfaces cannot be sharp. Nature Mater. 5, 204-209 (2006).

5. Thiel, S., Hammerl, G., Schmehl, A., Schneider, C. W. \& Mannhart, J. Tunable quasi-two-dimensional electron gases in oxide heterostructures. Science 313, 1942-1945 (2006).

6. Brinkman, A. et al. Magnetic effects at the interface between non-magnetic oxides. Nature Mater. 6, 493-496 (2007).

7. Reyren, N. et al. Superconducting interfaces between insulating oxides. Science 317, 1196-1199 (2007).

8. Caviglia, A. D. et al. Electric field control of the $\mathrm{LaAlO}_{3} / \mathrm{SrTiO}_{3}$ interface ground state. Nature 456, 624-627 (2008).

9. Singh-Bhalla, G. et al. Built-in and induced polarization across $\mathrm{LaAlO}_{3} / \mathrm{SrTiO}_{3}$ heterojunctions. Nature Phys. 7, 80-86 (2011).

10. Cen, C. et al. Nanoscale control of an interfacial metal-insulator transition at room temperature. Nature Mater. 7, 298-302 (2008).

11. Cen, C., Thiel, S., Mannhart, J. \& Levy, J. Oxide nanoelectronics on demand. Science 323, 1026-1030 (2009).

12. Xie, Y. W., Bell, C., Yajima, T., Hikita, Y. \& Hwang, H. Y. Charge writing at the $\mathrm{LaAlO}_{3} / \mathrm{SrTiO}_{3}$ surface. Nano Lett. 10, 2588-2591 (2010).

13. Xie, Y. W., Bell, C., Hikita, Y. \& Hwang, H. Y. Tuning the electron gas at an oxide heterointerface via free surface charges. Adv. Mater. 23, 1744-1747 (2011).
14. Pentcheva, R. et al. Parallel electron-hole bilayer conductivity from electronic interface reconstruction. Phys. Rev. Lett. 104, 166804 (2010).

15. Huijben, M. et al. High mobility interface electron gas by defect scavenging in a modulation doped oxide heterostructure (http://arxiv.org/abs/10081.896, 2010).

16. Siemons, W. et al. Origin of charge density at $\mathrm{LaAlO}_{3}$ and $\mathrm{SrTiO}_{3}$ heterointerfaces: possibility of intrinsic doping. Phys. Rev. Lett. 98, 196802 (2007)

17. Willmott, P. R. et al. Structural basis for the conducting interface between $\mathrm{LaAlO}_{3}$ and $\mathrm{SrTiO}_{3}$. Phys. Rev. Lett. 99, 155502 (2007).

18. Hwang, H. Y. Tuning interface states. Science. 313, 1895-1896 (2006)

19. Popović, Z. S., Satpathy, S. \& Martin, R. M Origin of the two-dimensional electron gas carrier density at the $\mathrm{LaAlO}_{3}$ and $\mathrm{SrTiO}_{3}$ interface. Phys. Rev. Lett. 101, 256801 (2008)

20. Pentcheva, R. \& Pickett, W. E. Avoiding the polarization catastrophe in $\mathrm{LaAlO}_{3}$ overlayers on $\mathrm{SrTiO}_{3}(001)$ through polar distortion. Phys. Rev. Lett. 102, 107602 (2009)

21. Son, W. J., Cho, E., Lee, J., \& Han, S. Hydrogen adsorption and carrier generation in $\mathrm{LaAlO}_{3}-\mathrm{SrTiO}_{3}$ heterointerfaces: a first-principles study. J. Phys.: Condens. Matter. 22, 315501 (2010).

22. Bristowe, N. C., Littlewood, P. B. \& Artacho, E. Surface defects and conduction in polar oxide heterostructures. Phys. Rev. B 83, 205405 (2011).

23. Bell, C et al. Dominant Mobility Modulation by the Electric Field Effect at the $\mathrm{LaAlO}_{3} / \mathrm{SrTiO}_{3}$ Interface. Phys. Rev. Lett. 103, 226802 (2009).

24. Feibelman, P. J. The first wetting layer on a solid. Phys. Today. 63, 34-39 (2010).

25. Jun, J. H., Kim, H. J. \& Choi, D. J. Effect of hydration on the properties of lanthanum oxide and lanthanum aluminate thin films. Ceram. Int. 34, 957-960 (2008).

26. Yan, B. D., Meilink, S. L., Warren, G. W. \& Wynblatt, P. Water adsorption and surface conductivity measurements on $\alpha$-alumina substrates. IEEE Trans. Compon., Hybrids, Manufact. Technol. 10, 247-251 (1987).

27. Eng, P. J. et al. Structure of the hydrated $\alpha-\mathrm{Al}_{2} \mathrm{O}_{3}(0001)$ surface. Science 288, 1029-1033 (2000).

28. Bi, F. et al. 'Water-cycle' mechanism for writing and erasing nanostructures at the $\mathrm{LaAlO}_{3} / \mathrm{SrTiO}_{3}$ interface. Appl. Phys. Lett. 97, 173110 (2010).

29. Ibbetson, J. P. et al. Polarization effects, surface states, and the source of electrons in $\mathrm{AlGaN} / \mathrm{GaN}$ heterostructure field effect transistors. Appl. Phys. Lett. 77, 250-252 (2000).

30. Schwingenschlögl, U. \& Schuster, C. Surface effects on oxide heterostructures. Europhys. Lett. 81, 17007 (2008).

31. Yoshimatsu, K., Yasuhara, R., Kumigashira, H. \& Oshima, M. Origin of metallic states at the heterointerface between the band insulators $\mathrm{LaAlO}_{3}$ and $\mathrm{SrTiO}_{3}$. Phys. Rev. Lett. 101, 026802 (2008).

32. Segal, Y., Ngai, J. H., Reiner, J. W., Walker, F. J. \& Ahn, C. H. X-ray photoemission studies of the metal-insulator transition in $\mathrm{LaAlO}_{3} / \mathrm{SrTiO}_{3}$ structures grown by molecular beam epitaxy. Phys. Rev. B 80, 241107 (2009).

33. Takizawa, M., Tsuda, S., Susaki, T., Hwang, H. Y. \& Fujimori, A. Electronic charges and electric potential at $\mathrm{LaAlO}_{3} / \mathrm{SrTiO}_{3}$ interfaces studied by core-level photoemission spectroscopy (http://arxiv.org/abs/1106.3619, 2011).

34. Ogawa, N. et al. Enhanced lattice polarization in $\mathrm{SrTiO}_{3} / \mathrm{LaAlO}_{3}$ superlattices measured using optical second harmonic generation. Phys. Rev. B 80, 081106 (2009).

\section{Acknowledgements}

We thank N. Ogawa for useful discussions, and K. Nishio, R. Takahashi and M. Lippmaa for technical assistance. Y.W.X. acknowledges funding from the Japan Society for the Promotion of Science and U.S. Air Force Office of Scientific Research (FAQSSO-10-10524). Y.H., C.B. and H.Y.H. acknowledge support by the Department of Energy, Office of Basic Energy Sciences, under Contract No. DE-AC02-76SF00515.

\section{Author contributions}

Y.W.X. performed sample fabrication, measurements and data analysis. Y.H., C.B. and H.Y.H. assisted with the planning, measurements and analysis.

\section{Additional information}

Competing financial interests: The authors declare no competing financial interests

Reprints and permission information is available online at http://npg.nature.com/ reprintsandpermissions/

How to cite this article: Xie Y. et al. Control of electronic conduction at an oxide heterointerface using surface polar adsorbates. Nat. Commun. 2:494 doi: 10.1038/ncomms1501 (2011) 